\title{
"A genetic defect of the mitochondrial energy supply: characterization of a novel
}

NDUFAF5 mutation"

${ }^{1}$ Itamar Raz, ${ }^{1}$ Aviram Kogot-Levin, ${ }^{2}$ Ann Saada and ${ }^{1}$ Sarah Weksler-Zangen

${ }^{1}$ Department of Internal Medicine, Diabetes Unit, Hadassah University Hospital, Jerusalem, Israel, E-mail: sarahz@hadassah.org.il

${ }^{2}$ Department of Human Genetics and Metabolic Diseases, Hadassah University Hospital, Jerusalem, Israel.

\section{Background}

Mitochondria is crucial for glucose stimulated insulin secretion (GSIS) by coupling glucose oxidation to ATP production. Increasing evidence established a role for mitochondrial dysfunction in diabetes. Cohen diabetic sensitive rats (CDs, Figure 1), a unique model of mitochondrial respiratory and complex-IV deficiency (Kogot-Levin A et al. Plos One 2016), develops hyperglycemia due to selective $\beta$ cell dysfunction when fed a diabetogenic high sucrose and copper deficient diet (HSD) but maintains normoglycemia on a regular diet (RD, weksler-zangen s. et al. Diabetes 2008, AJP 2013). Cytochrome $c$ Oxidase (COX, complex IV) is the terminal complex of the mitochondrial respiratory chain important for ATP production (Figure 2A) and consists of 13 individual subunits. Subunits 1-3, the catalytic core, are encoded in the mitochondrial genome and are copper dependent. Thus, copper is a key element for the catalytic activity of complex-IV. NDUFAF5 (NADH: Ubiquinone Oxidoreductase-Complex-Assembly-Factor-5) gene, encodes a mitochondrial complex I (Cl) assembly factor associated (Figure 2B) with $\mathrm{Cl}$ and IV deficiencies in Leigh-syndrome patients.

Our aim was to examine complex-I and complex-IV activity in islets and fibroblasts in relation to progression to diabetes in CDs-rats attempting to elucidate the genetic-basis of their $\beta$-cell dysfunction.

\section{Methods}

Eight weeks old diabetic prone $\mathrm{CDs}$ and non-diabetic control rats $(\mathrm{CDr})$, were fed a RD or a HSD for $\mathbf{3 0}$ days. Activity of complex IV normalized to the activity of the ubiquitous mitochondrial matrix enzyme, citrate-synthase (CS) was determined spectrophotometrically (figure 2A). Blood-glucose and insulinconcentrations were measured before and during OGTT performed at different periods on HSD or copper-supplemented-HSD. Whole-genome-sequencing was performed in blood-DNA using Illumina HiSeq2500 followed by in-silico variantanalysis. Protein-levels were determined by Western-blot. Rescue experiments were conducted by lentiviral-vector overexpression of a wild-type NDUFAF5gene, in CDs primary-fibroblasts

\section{Results}

We detected a progressive increase in blood glucose levels and a decrease in insulin secretion and islet-COX-activity with time on the HSD (Figures 3A-C). At 4-days on HSD, CDs-rats maintained normal-glucose-tolerance but insulin secretion and islet-COX-activity were significantly reduced. Impaired-glucosetolerance developed on day-11 when COX-activity was reduced to $56 \pm 0.6 \%$ and overt-hyperglycemia was observed at 20-days when COX-activity decreased to $37 \pm 6.8 \%$ ( $P<0.01$ vs CDs-RD). This deleterious process was reversed by feeding hyperglycemic-CDs-rats a copper-supplemented-HSD for 7-days, which increased COX-activity up-to $66 \pm 2.3 \%$ thereby restoring normoglycemia (Figures 3A-C). A highly significant positive correlation between islets and lymphocytes complex-IV activity and GSIS was observed. An inverse correlation between islets and lymphocytes complex-IV activity and blood-glucose-levels ( $<<0.0001$ respectively) was found in CDs fed HSD or copper-supplementedHSD. Accordingly, the minimal coX-activity (threshold) sustaining normoglycemia ranges between $56-66 \%$ (Figure 4).

cOX-activity \& complex-I activity were reduced in fibroblasts, heart, liver, islets and islets-mitochondria of CDs ( $P<0.01$ vs CDr, Figure $5 A$ and $B$ ). Protein levels of COX1 were significantly decreased in the different organs of CDs rats (Figure 5C). Whole-genome-sequencing identified a novel homozygous missense variant - p.P318L (c.C1002T), in the NDUFAF5 gene predicted to be highly pathogenic by in-silico tools. NDUFAF5 protein level was significantly decreased in CDs fibroblast \& islets compared to CDr (Figure 5D). Overexpression of wild-type NDUFAF5-gene in CDs-fibroblasts increased both complex-I and complex-IV activities $(P<0.01$. Figure $5 E)$.

\section{Summary and Conclusions}

We demonstrated a direct correlation between reduced islet-COX-activity, diminished GSIS and diabetes development. We identified islets COX-activity at the range of $56-66 \%$ of baseline as the metabolic threshold required to maintain normal glucose tolerance. The tight-correlation between COX-activity in islets and lymphocytes suggests that COX-activity in lymphocytes can serve as biomarker for $\beta$-cell COX-activity. Complex IV was initially reduced in islets of normoglycemic-CDs rats fed RD and was further decreased in islets of hyperglycemic-CDs rats fed the diabetogenic-diet, HSD. This suggests that impaired islet-mitochondrial function underlies the genetic-susceptibility of the CDs-rats to develop diabetes, positioning the cohen-rat as a model of mitochondrial disease-associated diabetes.

The tight correlation between impaired mitochondrial function and $\beta$-cell dysfunction in CDs rats, suggest that it is a model of mitochondrial-disorder related diabetes. The identified novel homozygous missense variant, p.P318L in the NDUFAF5-gene, may underlie the CDs-mitochondrial defect and susceptibility to develop diabetes.

\section{Figure 1: The CD rat model phenotype}

\section{The CD rat Model Phenotypes}
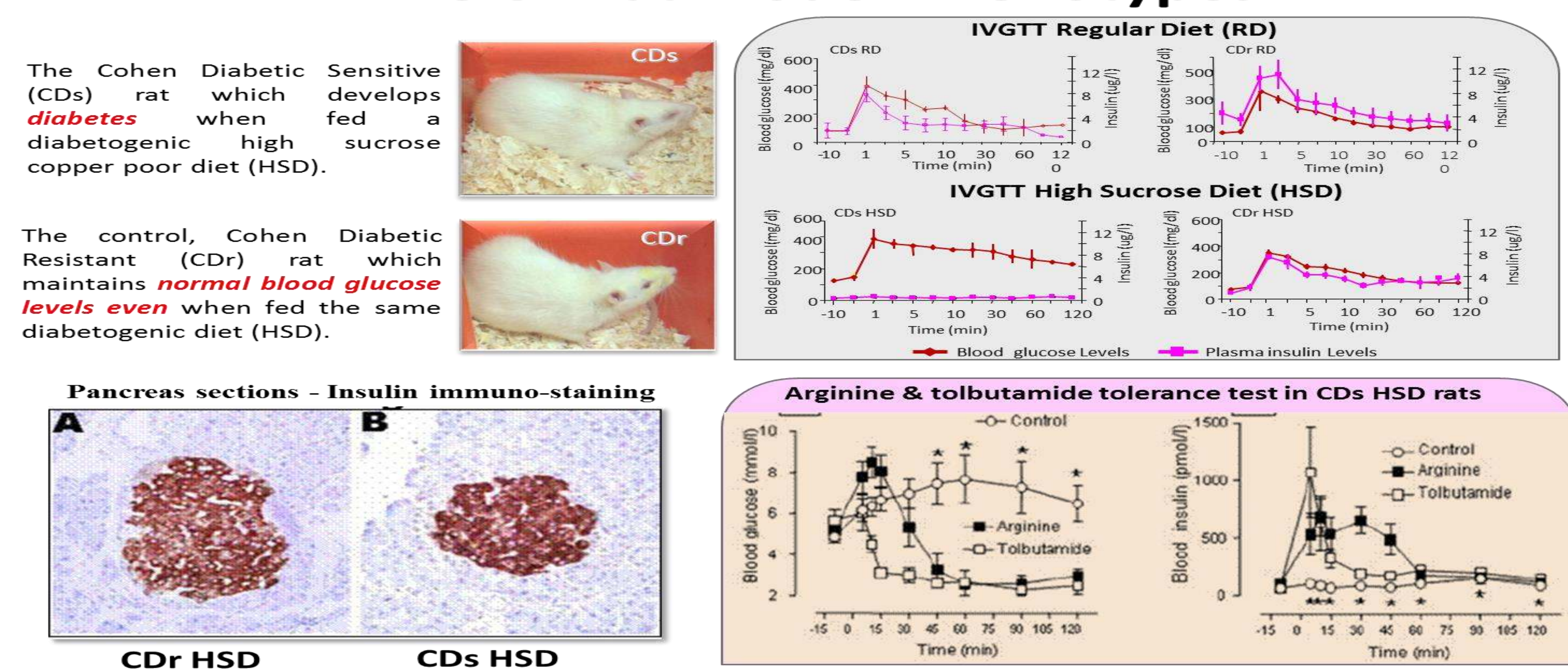

Fig 1. A- The Cohen diabetic rat model. B- intravenous glucose tolerance test (IVGTT) on CDs and CDr rats fed RD and HSD. CDs rats fed HSD exhibit markedly increased blood glucose levels and blunted GSIS when fed HSD (Weksler-Zangen S. Diabetes

Figure 2A-B: Mitochondrial enzymes and assembly factors

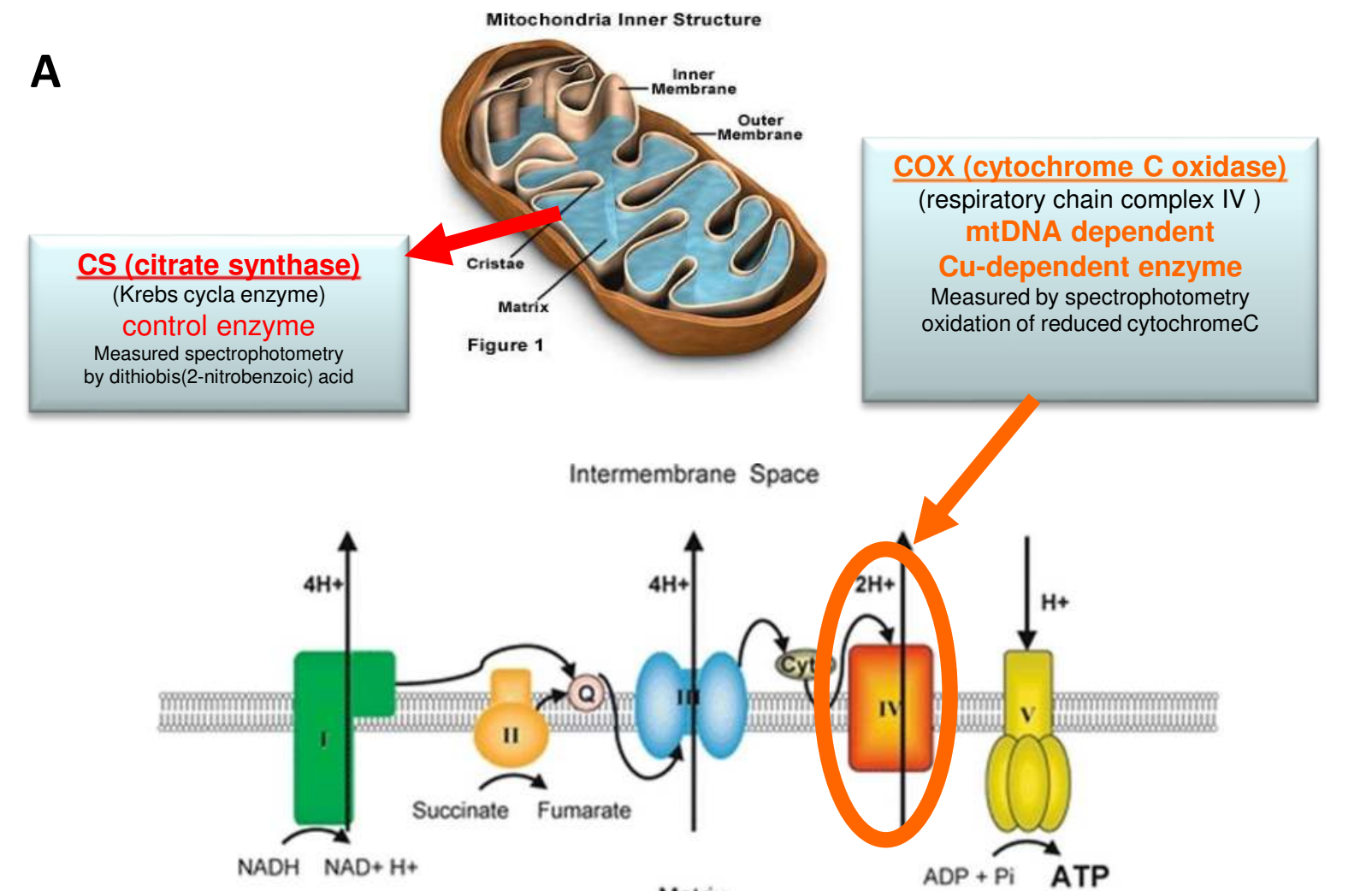

Participation of NDUFAF5 in the pathway of assembly of human complex I

Figure 3A-E: Blood glucose, insulin and COX activity
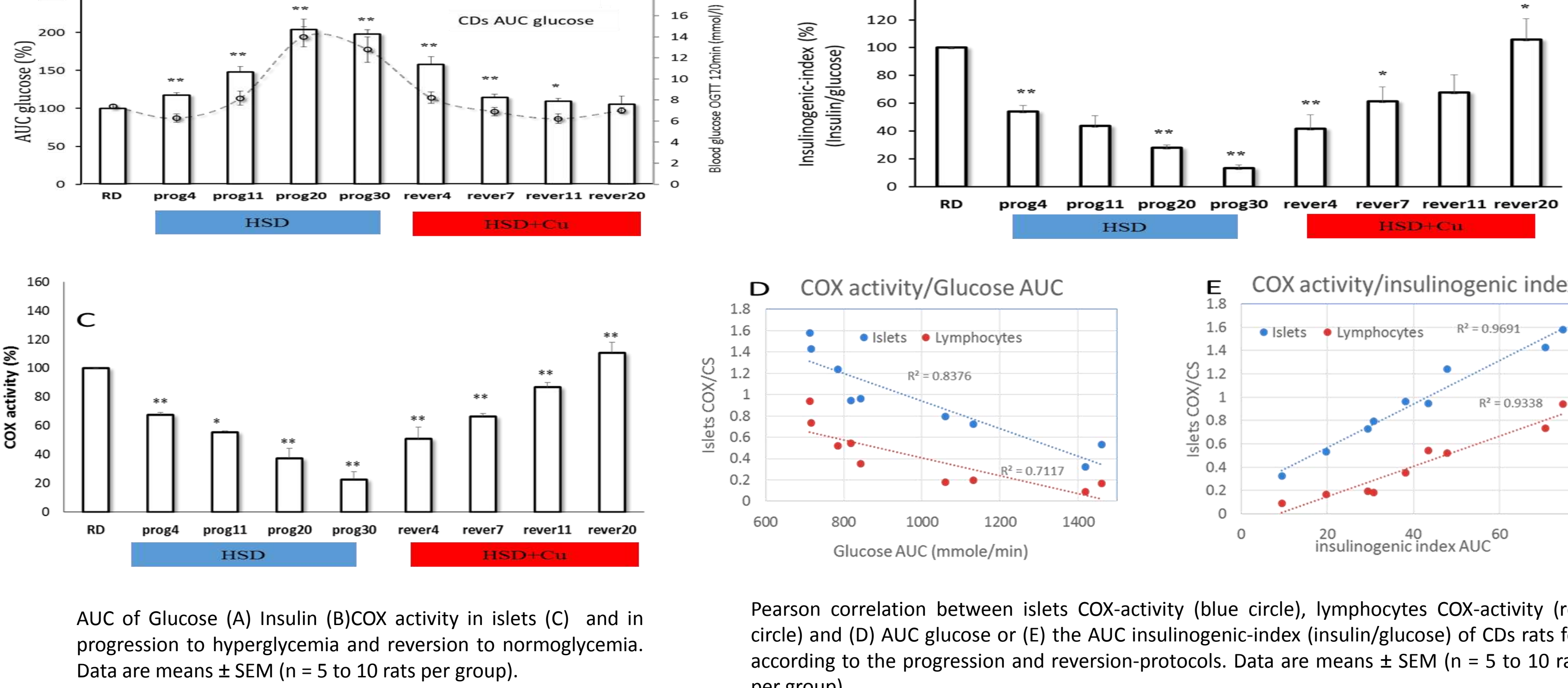

\section{Figure 4: COX threshold}

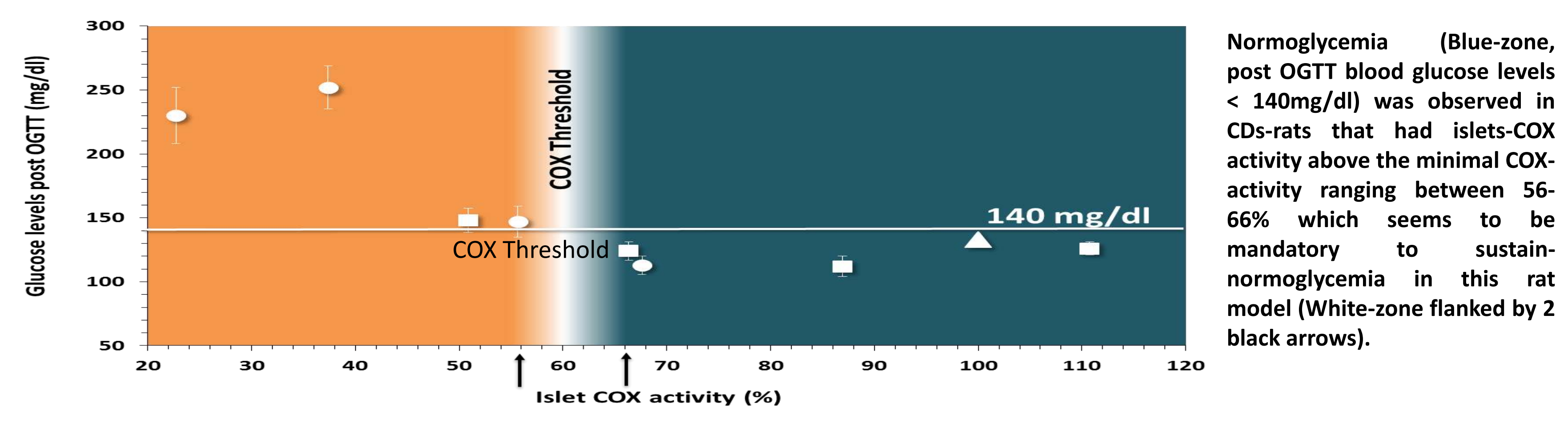

Figure 5A - 5E: NADUFAF5

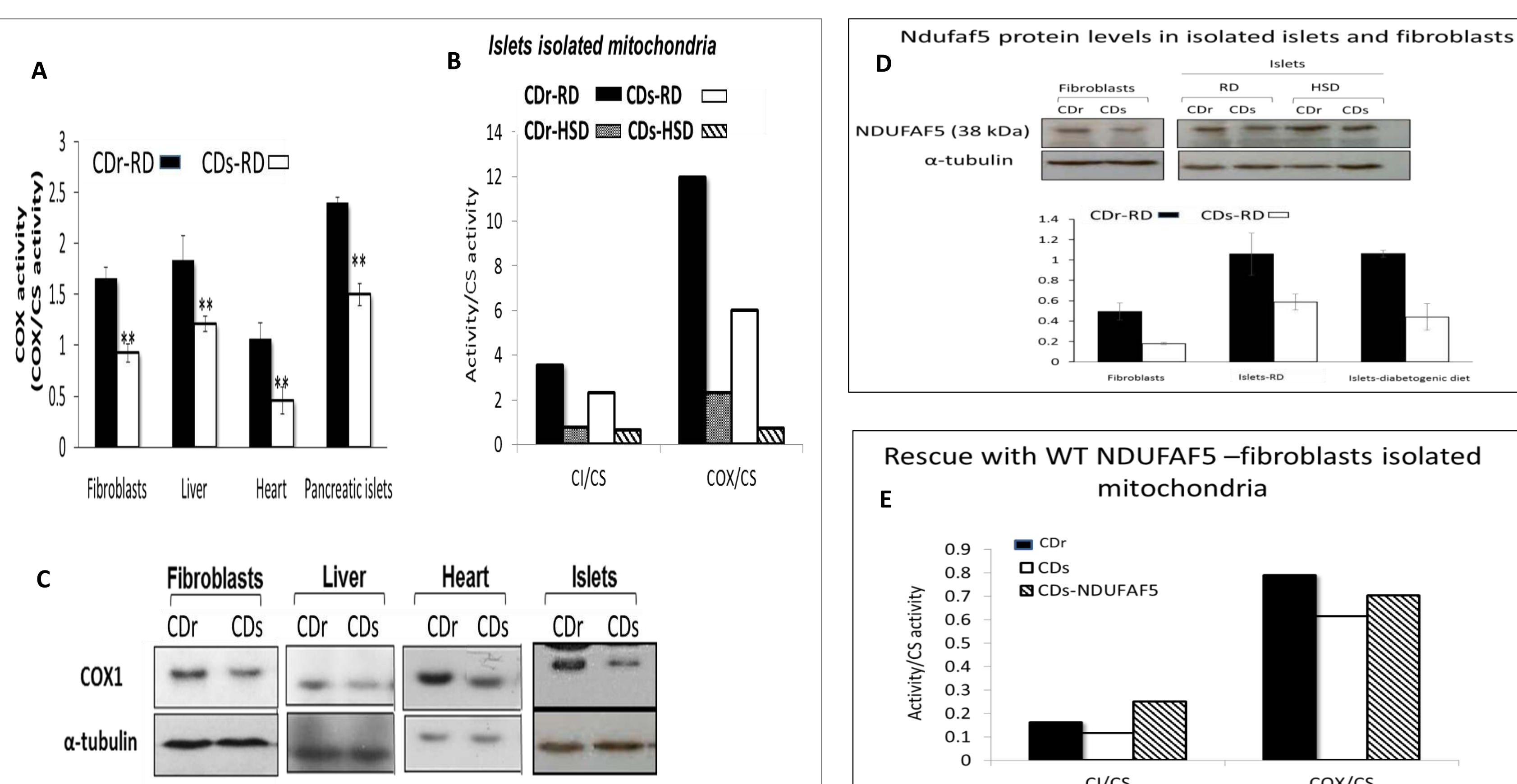

